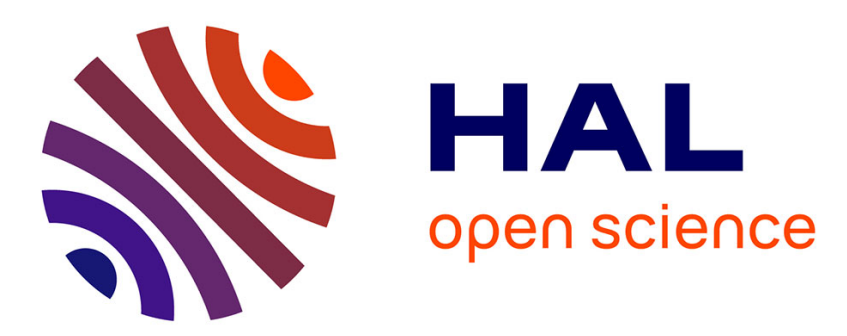

\title{
Theoretical Study of the Gas-Phase Thermal Decomposition of Urea
}

\author{
J. Honorien, R. Fournet, P.-A. Glaude, B. Sirjean
}

\section{To cite this version:}

J. Honorien, R. Fournet, P.-A. Glaude, B. Sirjean. Theoretical Study of the Gas-Phase Thermal Decomposition of Urea. Proceedings of the Combustion Institute, 2021, 38 (1), pp.355-364. 10.1016/j.proci.2020.06.012 . hal-03357408

\section{HAL Id: hal-03357408 \\ https://hal.science/hal-03357408}

Submitted on 28 Sep 2021

HAL is a multi-disciplinary open access archive for the deposit and dissemination of scientific research documents, whether they are published or not. The documents may come from teaching and research institutions in France or abroad, or from public or private research centers.
L'archive ouverte pluridisciplinaire HAL, est destinée au dépôt et à la diffusion de documents scientifiques de niveau recherche, publiés ou non, émanant des établissements d'enseignement et de recherche français ou étrangers, des laboratoires publics ou privés. 


\title{
Theoretical Study of the Gas-Phase Thermal Decomposition of Urea
}

\author{
J. Honorien ${ }^{1}$, R. Fournet ${ }^{1}$, P.-A. Glaude ${ }^{1}$, B. Sirjean ${ }^{1, *}$ \\ ${ }^{1}$ Laboratoire Réactions et Génie des Procédés, CNRS, Université de Lorraine \\ 1 rue Grandville BP 2045154001 Nancy Cedex, France
}

Corresponding author :

Baptiste Sirjean

Laboratoire Réactions et Génie des Procédés

1 rue Grandville BP 2045154001 Nancy Cedex,

France

Email: baptiste.sirjean@univ-lorraine.fr

Word count:

Main text: 3947 words

Table $1(5+2) * 7.6 * 2+46=152$

Table $2(2+2) * 7.6 * 2+48=108$

Figure $1(39+10) * 2.2+21=129$

Figure $2(54+10) * 2.2+23=164$

Figure $3(35+10) * 2.2+49=148$

Figure $4(65+10) * 2.2+22=187$

Figure $5(44+10) * 2.2+23=164$

Figure $6(35+10) * 2.2+26=125$

Figure $7(38+10) * 2.2+42=148$

References $(16+2) * 2.3 * 7.6=349$

Total: 5620 


\section{Abstract}

Urea is used in Selective Catalytic and Non Catalytic Reduction (SCR and SNCR) methods for NOx abatement in post-combustion processes. The decomposition of urea-water solution in exhaust systems leads to the formation of $\mathrm{NH}_{3}$, which is the $\mathrm{NOx}$ reducing agent. However, the decomposition of urea can also lead to the formation of unwanted pyrolysis by-products, particularly in region where the temperature and pressure conditions are not optimal or via wall-spray interactions. In this study, a gas phase kinetic model for urea pyrolysis is proposed to explain the growth of pyrolysis by-products of urea, and in particular, the route of formation of the major product: isocyanuric acid. Systematic theoretical calculations, using electronic structure calculations and transition state theory, were performed to explore all the possible unimolecular and bimolecular reactions of the initial species. Kinetic modeling was used to select the relevant reaction pathways at each growing step, and their rate constants were refined using CCSD(T)/CBS//B2PLYP-D3/cc-pvTZ calculations. Quantum calculations showed that the postulated growing schemes of the literature, based on the successive urea + $\mathrm{HNCO} \leftrightarrows$ biuret, biuret $+\mathrm{HNCO} \leftrightarrows$ triuret followed by the cyclization of triuret into isocyanuric acid are not energetically favored. It is observed that the most favored reaction route to isocyanuric acid involves carbamimidic acid, the urea tautomer, that first yields biuret in a reaction with $\mathrm{HNCO}$ and then is involved in the formation of triuret by reacting with a decomposition product of biuret. Finally, isocyanuric acid is formed from a decomposition product of triuret. This new mechanism of formation of isocyanuric acid is tested at different conditions to explore its most favored conditions of formation in the gas phase. Exploratory simulations of a pseudo condensed phase are also performed to qualitatively simulate condensed phase experiments and explain the observed pyrolysis yields. 


\section{Introduction}

Selective Catalytic Reduction (SCR) and Selective Non Catalytic Reduction (SNCR) are the most commonly used methods for $\mathrm{NOx}$ abatement in post-combustion treatments [1]. $\mathrm{NH}_{3}$ is the reducing agent in these processes, but because of safety hazards associated with its storage and manipulation in Diesel vehicles, urea is used as an ammonia reservoir. The decomposition of a urea-water solution (32.5 wt\% urea in water) yields ammonia and HNCO. Then, $\mathrm{HNCO}$ reacts with water to form $\mathrm{NH}_{3}$ and $\mathrm{CO}_{2} \cdot \mathrm{NO}_{\mathrm{x}}$ removal in urea based SNCR is possible in a temperature range of $\approx 1150$ to $1450 \mathrm{~K}$ [1]. The presence of a catalyst in ureaSCR processes leads to lower operating temperatures $(\approx 500-700 \mathrm{~K})$. In these processes, the water-urea solution is injected in the exhaust pipe and undesired byproducts can be produced in temperature zones below the optimal operating temperatures, because urea decomposition starts at $400 \mathrm{~K}$ [2]. Spray-wall interaction is a well-known source of undesired byproducts of urea thermal decomposition that leads to liquid and solid deposits that can degrade the selective reduction processes [2].

Stein et al. showed that the evaporation and decomposition of a droplet of urea-water solution occurs in three main phases: water evaporation, urea decomposition and reactions in the gas phase [3]. Thus, there is a need to understand the mechanism of urea thermal decomposition and the formation routes of the pyrolysis products. Many studies of the literature proposed qualitative or semi-quantitative reaction mechanisms based on experimental results, mostly obtained using Thermogravimetric Analysis (TGA) and/or Differential Scanning Calorimetry (DSC) methods coupled with analytical systems [2, 4-8]. All these studies, performed between 400 and $900 \mathrm{~K}$, showed that beside the desired products of urea thermal decomposition $\left(\mathrm{NH}_{3}\right.$ and $\left.\mathrm{HNCO}\right)$, the following byproducts are measured: biuret, triuret, ammelide, ammeline, melamine and cyanuric acid. The latter compound is found to be the major byproduct between 473 and $623 \mathrm{~K}[4,7,8]$. It is not surprising since the industrial 
production of cyanuric acid involves the thermal decomposition of urea [9]. Analyses of the light compounds showed that $\mathrm{NH}_{3}, \mathrm{HNCO}$ and $\mathrm{CO}_{2}$ are also produced in large quantities.

Several reaction mechanisms were proposed in these studies to explain the formation of the pyrolysis products of urea. The latest kinetic mechanism of the literature, based on literature review and simulations of TGA experiments [2] can be summarized as follow:

$$
\begin{aligned}
& \text { urea } \leftrightarrows \mathrm{NH}_{3}+\mathrm{HNCO} \\
& \text { urea }+\mathrm{HNCO} \leftrightarrows \text { biuret } \\
& \text { biuret }+\mathrm{HNCO} \leftrightarrows \text { triuret } \\
& \text { triuret } \leftrightarrows \text { cyanuric acid }+\mathrm{NH}_{3} \\
& \text { triuret } \leftrightarrows \text { ammelide }+\mathrm{H}_{2} \mathrm{O}
\end{aligned}
$$

Following the unimolecular decomposition of urea (R1), HNCO reacts with urea (R2) to form biuret, which can in turn reacts with HNCO to produce triuret. The cyclization of triuret leads to the formation of cyanuric acid (R4) and ammelide (R5). In recent TGA experiments, Wang et al.[8] concluded that biuret and triuret were formed from the self-reaction of urea and the reaction of urea with biuret, respectively. The simulations of Tischer et al. [2] included these reactions in their kinetic mechanism.

$$
\begin{array}{r}
\text { urea }+ \text { urea } \leftrightarrows \text { biuret }+\mathrm{NH}_{3} \\
\text { biuret }+ \text { urea } \leftrightarrows \text { triuret }+\mathrm{NH}_{3}
\end{array}
$$

To the best of our knowledge, no theoretical study has attempted to explore the formation routes of the byproducts of urea pyrolysis. Nicolle et al. [10] performed a modeling study of urea decomposition kinetics in solution, based on electronic structure calculations and implicit solvent methods. The initial unimolecular decomposition of urea in water was found to yield $\mathrm{NH}_{3}$ and HCNO. Gratzfeld and Olzmann [11] calculated the enthalpy of formation of all the urea-derived compounds involved in reactions R1 to R7 using high-level CCSD(T)-F12 calculations. 
This study aims to unravel the formation routes of the major pyrolysis products of urea using the tools of theoretical chemical kinetics. Experiments on urea thermal decomposition are heterogeneous systems, where the reactions in the different states of matter are not known. This work focuses on the gas phase to establish the reaction mechanism in this state. It will help in the interpretation of the literature data by allowing the decoupling of the intricate gazliquid-solid data. In SCR and SNCR processes, it is a droplet of liquid urea that is subjected to pyrolysis [3]. The proposed gas-phase kinetic mechanism will be able to serve as a starting point in the development of a liquid phase kinetic model for the thermal decomposition of urea. These models are based on the gas phase reactions and their thermokinetic data are calculated from the ideal gas data and corrected by free energies of solvation [12, 13].

\section{Computational methods}

A large number of reactions was investigated in this study because of the possible combinations in the bimolecular reactions. Because of the size of the heavier products, we first systematically explored the potential energy surfaces of all potential reaction pathways at the CBS-QB3 level of theory. The rates of formation of the pyrolysis byproducts involve bimolecular reactions and therefore depends on the mole fractions of the molecules in the system. Thus, a trial primary detailed kinetic model was developed based on the CBS-QB3 potential energy surfaces, with thermodynamic and kinetic data calculated using the harmonic oscillator approximation in statistical thermodynamic and transition state theory equations (with the ThermRot code [14]). Simulations were performed with the trial mechanism in a homogeneous batch reactor (using Chemkin-Pro software [15]) to select the relevant decomposition pathways. The thermokinetic data of the selected decomposition routes were then computed at a higher level of calculation. Geometry optimization and frequency calculations were performed at the B2PLYP-D3/cc-pVTZ level of theory. Single point calculations were made at the $\operatorname{CCSD}(\mathrm{T})$ level of calculation with cc-pVDZ and cc-pVTZ basis sets and at the MP2 level with cc-pVQZ, cc-pvTZ and cc-pVQZ basis sets. Complete 
basis set extrapolation (CBS) was done using the single point calculations at the $\operatorname{CCSD}(\mathrm{T})$ and MP2 levels, using the equations reported by Miller and Klippenstein [16]. This level of calculation will be denoted $\operatorname{CCSD}(\mathrm{T}) / \mathrm{CBS} / / \mathrm{B} 2 \mathrm{PLYP}-\mathrm{D} 3 / \mathrm{cc}-\mathrm{pVTZ}$ in the text. Internal rotations were systematically treated using the uncoupled 1D-HR (1D-HRU) method implemented in the ThermRot software, based on relaxed scans performed at the B2PLYPD3/cc-pVTZ level of theory. Quantum tunneling was considered for H-atom transfer reactions using the Eckart potential approach.

The final detailed kinetic model of urea pyrolysis includes the selected reaction pathways and a reaction basis from Glarborg et al. [17]. This reaction basis includes the combustion reactions of $\mathrm{C}_{1}-\mathrm{C}_{2}$ hydrocarbons, amines, cyanides and hydrocarbon/nitrogen interactions. Simulations were performed in a homogeneous batch reactor for the gas phase pyrolysis of pure urea.

\section{Computational results}

\subsection{Unimolecular decomposition of urea}

Figure 1 presents the potential energy surface of urea unimolecular decomposition.

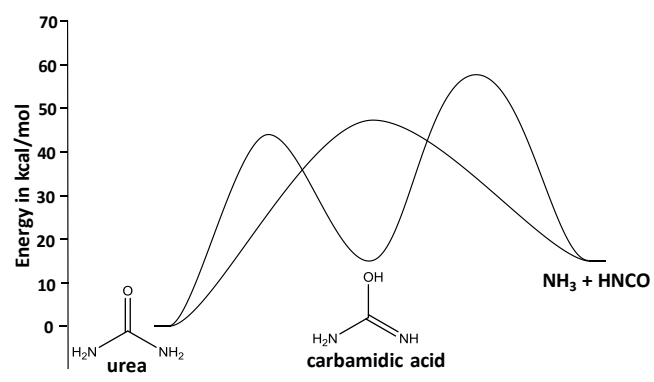

Figure 1: Potential energy surface of the unimolecular decomposition of urea. Energy at $0 \mathrm{~K}$ computed at the CCSD(T)/CBS//B2PLYP-D3/cc-pVTZ level of theory.

The formation of $\mathrm{NH}_{3}$ and $\mathrm{HNCO}$ from urea is possible through the direct elimination via a 4membered transition state (TS) structure $(48.5 \mathrm{kcal} / \mathrm{mol}$ energy barrier) or involves an internal $\mathrm{H}$-atom transfer yielding first carbamimidic acid (CA) (43.8 kcal/mol energy barrier), which in turn can eliminate $\mathrm{NH}_{3}$ and $\mathrm{HNCO}$ via a 4-membered ring TS. The decomposition of carbamimidic acid (CA) into $\mathrm{NH}_{3}$ involves a barrier of $43,3 \mathrm{kcal} / \mathrm{mol}$, so its reverse 
tautomerization to urea, with a barrier of $29.3 \mathrm{kcal} / \mathrm{mol}$, is more favored. These reactions were include in the trial mechanism as a starting point. It can be noted that the lowest bond dissociation energies of urea and CA are $97.8 \mathrm{kcal} / \mathrm{mol}(\mathrm{C}-\mathrm{N}$ bond) and $104 \mathrm{kcal} / \mathrm{mol}(\mathrm{O}-\mathrm{H}$ bond) at the CBS-QB3 level, respectively. Radical pathways will not be competitive with the molecular reactions. At equilibrium, for a given temperature and pressure, the system is composed of urea, $\mathrm{CA}, \mathrm{HNCO}$ and $\mathrm{NH}_{3}$. The growth of molecular structures towards the formation of biuret was explored by considering all the possible bimolecular reactions in the system.

\subsection{Formation of biuret in bimolecular reactions}

Table 1 presents all the possible bimolecular reactions envisaged in this study and the associated lowest energy barriers computed at the CBS-QB3 level of theory.

Table 1: Bimolecular reactions with the lowest energy barriers from the initial products of urea unimolecular decomposition. Energy barriers ( $\mathrm{kcal} / \mathrm{mol}$ ) at $0 \mathrm{~K}$ computed at the CBS-QB3 level of theory. 2D structures of reactions and energy barriers computed for higher energy reactions are given in Table S1.

\begin{tabular}{ccccc}
\hline & urea & CA & HNCO & NH3 \\
\hline urea & 35.9 & 20.5 & 15.2 & - \\
CA & - & 27.7 & -2.6 & 47.7 \\
HNCO & - & - & 36.8 & 33.1 \\
NH3 & - & - & - & - \\
\hline
\end{tabular}

A total of 24 reactions were investigated. For example, two different reactions were found for urea + urea, one leading to biuret $+\mathrm{NH}_{3}$, with a $51.1 \mathrm{kcal} / \mathrm{mol}$ energy barrier, and the other one yielding 1-[diamino(hydroxy)methyl]urea (see Table S1) with a barrier of $35.9 \mathrm{kcal} / \mathrm{mol}$. Given the energy barriers of other possible bimolecular reactions, it can already be concluded that reaction R6 is not the main formation route of biuret. It can be observed that the reactions of carbamimidic acid with HNCO is characterized by a very low negative energy barrier, which is explained by the formation of a Van der Waals complex between the reactants. The most favored reaction involving urea is the addition of $\mathrm{HNCO}(15.2 \mathrm{kcal} / \mathrm{mol}$ energy barrier $)$ that leads to the formation of carbamimidoyl carbamate (CC): 


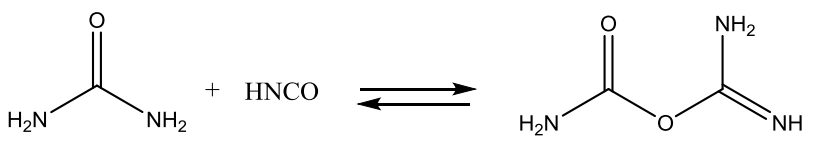

This reaction is favored by $20.2 \mathrm{kcal} / \mathrm{mol}$ over the formation of biuret postulated in the literature (reaction (R2), see Table S1).

Urea and CA can react through a 6-membered cyclic TS structure, with a $20.5 \mathrm{kcal} / \mathrm{mol}$ energy barrier, yielding the same product as in reaction (R9) (CC), and ammonia:<smiles>C=CNC(=N)OC(N)=O</smiles>

The lowest energy reaction of the bimolecular reaction matrix is the reaction between CA and HNCO that leads to the formation of biuret. Figure 2 depicts the potential energy surface for this reaction.

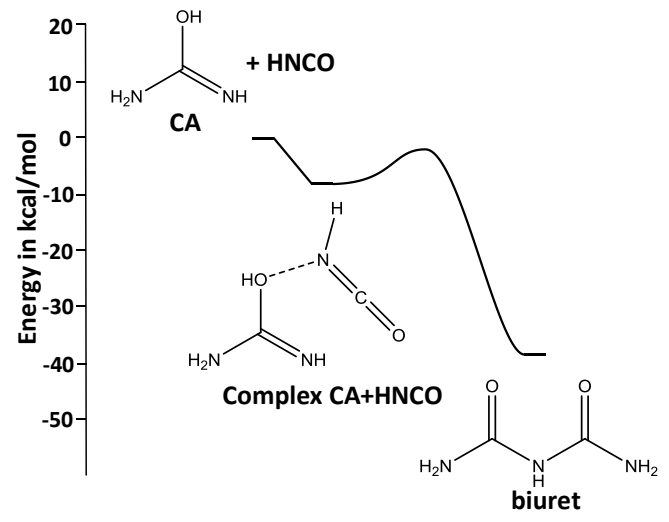

Figure 2: Potential energy surface of the carbamimidic acid (CA) + HNCO reaction. Energy at 0K computed at the CBS-QB3 level of theory.

The formation of biuret is favoured by the formation of a VdW complex that lies $8.4 \mathrm{kcal} / \mathrm{mol}$ below the entrance channel.

All the possible reactions of Table S1 were included in the trial detailed kinetic mechanism, with exit channels set as irreversible to determine the high limit of the product formation fluxes. Temperatures between 500 and $1500 \mathrm{~K}$ were investigated at atmospheric pressure. As a test case, a pressure of $1000 \mathrm{~atm}$ was also investigated to maximize the bimolecular rates and to emulate a condensed phase system. Figure S1 depicts the branching ratios as a function 
of temperature for the two pressures considered. The trial simulation shows that, beside the unimolecular decomposition pathways of urea (Figure 1), four bimolecular reactions can play a role in urea pyrolysis. At low temperature (below 600K at 1 atm and below $1000 \mathrm{~K}$ at 1000 atm), the formation of $\mathrm{CC}$ through reaction $\mathrm{R} 9$ is a major consumption route of urea and a major source of $\mathrm{NH}_{3}$. At atmospheric and 1000 atm pressures biuret is the major pyrolysis product from the reaction CA + HNCO (Figure S1), at temperatures above $600 \mathrm{~K}$ and $900 \mathrm{~K}$, respectively. For both pressures investigated, the formation of CG (Figure S1) is also observed, but with a small ratio. At atmospheric pressure and high temperature (above 1200 $\mathrm{K})$ the trial mechanism predicts that $\mathrm{HNCO}+\mathrm{HNCO}=>$ carbamic isocyanate $(36.8 \mathrm{kcal} / \mathrm{mol}$ energy barrier in Table 1) becomes a major product.

The four selected pathways lead to the formation of four new compounds: biuret, carbamimidoyl carbamate (CC), carbamoyl guanidine (CG) and carbamic isocyanate (CI). We therefore systematically explored the unimolecular decomposition routes of these species and included them in the trial mechanism. The possible bimolecular reactions of these four new compounds were also added in a second trial mechanism.

\subsection{Unimolecular decomposition of biuret and carbamoyl guanidine (CG)}

Biuret and CG are tautomeric structures that are interconnected. The possible isomerizations of these two structures lead to six interconnected tautomers. Figure S2 presents the six tautomers of biuret and the associated energy barriers for their isomerizations. Theoretical calculations show that from biuret and CG, only one tautomer (Biuret Tautomer, BT) can be formed with a favourable energy barrier. The remaining tautomers are too high in energy to play a role. Figure 3 presents the potential energy surface of biuret, CG and BT isomerization and decomposition. 


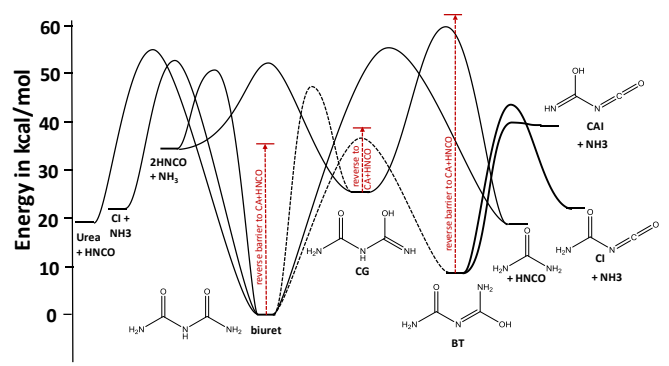

Figure 3 : Potential energy surface of biuret, CG and BT unimolecular decomposition. Energy at 0K computed at the CBS-QB3 level of theory. Red dashed lines represent the energy barriers for the reverse decomposition of biuret and its tautomers to CA and HNCO. Black dashed lines are tautomeric interconversions.

Four unimolecular pericyclic decomposition pathways are identified for biuret. The channels leading to urea $+\mathrm{HNCO}$ and $\mathrm{CA}+\mathrm{HNCO}$ are the reverse reactions of the growth mechanism of urea presented in Table 1. The two other decomposition routes involved the formation of $\mathrm{CI}+\mathrm{NH}_{3}$ and $2 \mathrm{HNCO}+\mathrm{NH}_{3}$. The reverse dissociation of biuret to $\mathrm{CA}$ and $\mathrm{HNCO}$ has the lowest energy barrier $(35.5 \mathrm{kcal} / \mathrm{mol})$ that lies, at least, $15.7 \mathrm{kcal} / \mathrm{mol}$ below the critical energies of other decomposition channels. The only elementary mechanism that can compete with the reverse dissociation is the isomerization to the BT tautomer, that features a critical energy of $37.1 \mathrm{kcal} / \mathrm{mol}$. For the CG tautomer of biuret, no decomposition route can compete with the reverse dissociation to $\mathrm{CA}+\mathrm{HNCO}$ that has an energy barrier of $13.7 \mathrm{kcal} / \mathrm{mol}$. Such a low critical energy indicates that CG is not stable at pyrolysis temperatures. The BT tautomer is found to be more stable as its reverse dissociation to CA + HNCO features an energy barrier of $61.9 \mathrm{kcal} / \mathrm{mol}$. CBS-QB3 calculations show that the decomposition of BT into CI and its tautomer in its carbamimidic acid form (CAI) represent the lowest energies decomposition channels that can compete with the reverse dissociation of biuret

\subsection{Unimolecular decomposition of carbamimidoyl carbamate (CC) and carbamic isocyanate isomers (CI and CAI)}

The most favoured unimolecular decomposition of CI and CAI are their reverse dissociations to $2 \mathrm{HNCO}$ which face energy barriers of 48.4 and $42.9 \mathrm{kcal} / \mathrm{mol}$, respectively. The isomerization reaction between $\mathrm{CI}$ and $\mathrm{CAI}$ 


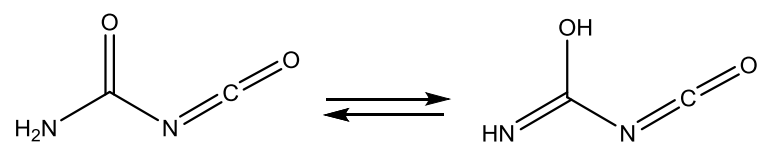

features a critical energy of $48.5 \mathrm{kcal} / \mathrm{mol}$ and was included in the second trial mechanism.

CC can decompose into two sets of products in reaction (R11) and (R12):<smiles>CCN=C=[NH+]NC(=O)O</smiles>

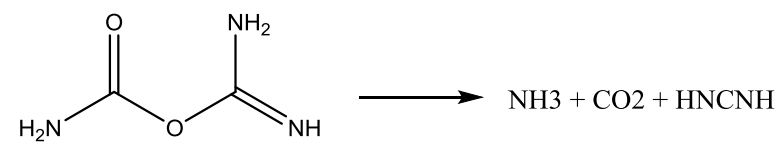

Reaction R11 involves a 6-membered TS structure and features the most favourable energy barrier $(28.5 \mathrm{kcal} / \mathrm{mol})$ compared to reaction R12 (39.9 kcal/mol). NHCNH (carbodiimide) and $\mathrm{NH}_{2} \mathrm{COOH}$ (carbamic acid) are therefore expected to be easily formed from $\mathrm{CC}$ that will not be stable at pyrolysis temperatures.

\subsection{Bimolecular reactions of biuret, CI and CAI}

The unimolecular decomposition of $\mathrm{CG}$ and $\mathrm{CC}$ involve low energy barriers and these compounds will readily decompose at temperatures of interest. Biuret, CI and CAI are stable enough to be involved in bimolecular reactions with the molecules present in the system. These molecules include urea, $\mathrm{CA}, \mathrm{HNCO}$ and $\mathrm{NH}_{3}$. Biuret has similar reactive sites as urea and we can hypothesize that bimolecular reactions with itself, with CA, with $\mathrm{HNCO}$ and with urea involve similar energy barriers that urea and these molecules (Table 1, first row). These bimolecular processes were therefore written based on analogies with urea in the second trial kinetic model. Table 2 presents the lowest critical energies computed for the reactions of CI and $\mathrm{CAI}$ with themselves, $\mathrm{CA}, \mathrm{HNCO}$ and $\mathrm{NH}_{3}$.

Table 2: Lowest energy barriers of the bimolecular reactions of CI and CAI with the initial products of urea unimolecular decomposition. Energy barriers (kcal/mol) at $0 \mathrm{~K}$ computed at the CBS-QB3 level of theory. 2D structures of reactions and energy barriers computed for higher energy reactions are given in Table S2.

\begin{tabular}{cccccc}
\hline & CI & CAI & CA & HNCO & NH3 \\
\hline CI & 27.4 & 18.2 & -4.8 & 35.4 & 30.7 \\
\hline CAI & 18.2 & 21.2 & -4.1 & 10.9 & 0.2 \\
\hline
\end{tabular}


All the possible bimolecular reactions explored at the CBS-QB3 level of theory are given in Table S2. The reaction between CI and HNCO is part of the polymerization process of HNCO: $\mathrm{HNCO}+\mathrm{HNCO} \leftrightarrows \mathrm{CI}, \mathrm{CI}+\mathrm{HNCO} \leftrightarrows$ Allophanamic isocyanate (AI). CI+HNCO faces an energy barrier of $35.4 \mathrm{kcal} \mathrm{mol}^{-1}$, while the reaction of carbamimidic acid isocyanate (CAI) with HNCO has a lower energy barrier of $10.9 \mathrm{kcal} \mathrm{mol}^{-1}$. The latter reaction directly yields a tautomer of iso-cyanuric acid:

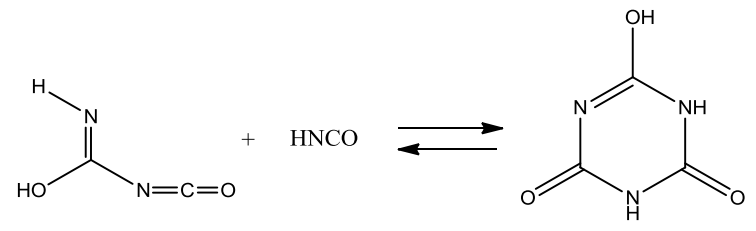

From Table 2, it can be seen that the reactions of CI and CAI with CA involve negative energy barriers, indicating the existence of pre-reactive Van der Waals complexes. Figure 4 depicts the potential energy surface of the reaction between CI and CA.

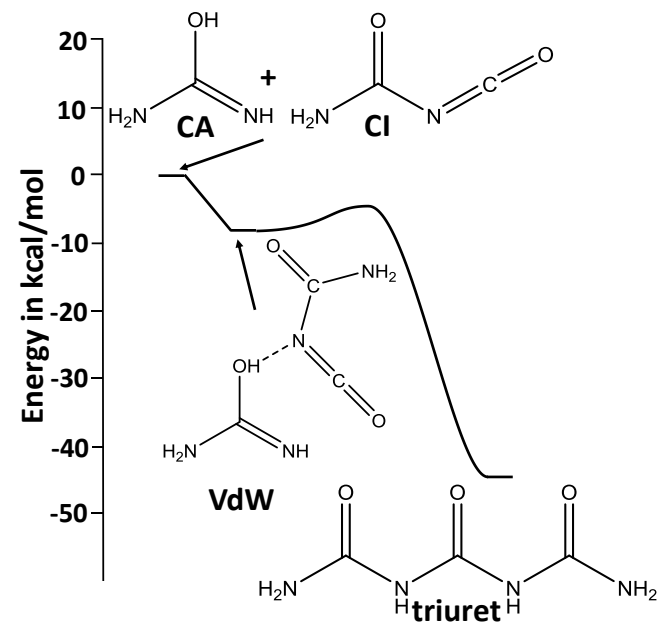

Figure 4: Potential energy surface of the reaction CA + CI, computed at the CBS-QB3 level of theory. Energy at $0 \mathrm{~K}$.

The reaction of CA with $\mathrm{CI}$ leads to the formation of a pre-reactive complex, characterized by a hydrogen bond between the $\mathrm{H}$-atom of the $\mathrm{OH}$ group of $\mathrm{CA}$ and the $\mathrm{N}$-atom of CI. From this Van der Waals complex, triuret is formed with an energy barrier lying below the entrance 
channel. A similar PES was obtained for the reaction of CAI with CA, yielding a tautomer of triuret (TriuretIminol in Table S2). Our calculations show that triuret is not formed from the reaction of biuret with $\mathrm{HNCO}(\mathrm{R} 3)$ or urea with biuret (R7), as assumed in the literature.

Based on the unimolecular decomposition routes of biuret, $\mathrm{CG}, \mathrm{CC}$, and $\mathrm{CI}$ as well as the bimolecular reactions of biuret, CI and CAI with the molecules of the system, a second trial kinetic mechanism was developed, with irreversible exit channels, and simulations were performed under the same conditions as the ones used for the first trial model. Figure S3 shows that $\mathrm{NH}_{3}$ yields is dominant over all the conditions studied. At low temperatures, $\mathrm{HNCNH}$ and $\mathrm{NH}_{2} \mathrm{COOH}$ formation is favoured. These products come from the unimolecular decomposition of $\mathrm{CC}$, and their formation is favoured over a larger range of temperature at high pressure. At atmospheric pressure, the formation of triuret from the reaction of CI and CAI with CA has a high branching ratio at intermediate temperatures. When the temperature reaches $1150 \mathrm{~K}$, HNCO formation becomes dominant. High-pressure simulations lead to the same conclusions but the stability of the intermediate products is shifted towards higher temperatures. In the following section, the unimolecular decomposition of triuret and carbamic acid are described. HNCNH decomposition reactions are already included in the core reaction mechanism., except for its polymerization with itself and with $\mathrm{HNCO}$ that were computed in this study.

\subsection{Unimolecular decomposition of triuret and carbamic acid}

Triuret can follow the same decomposition patterns as biuret, but with a larger number of tautomers. Its size is large enough to allow its cyclization into iso-cyanuric acid, the major byproduct of urea pyrolysis, as assumed in all literature studies (reaction (R4)). This reaction also yields $\mathrm{NH}_{3}$ and features a critical energy of $60.6 \mathrm{kcal} / \mathrm{mol}$, which lies $20.8 \mathrm{kcal} / \mathrm{mol}$ above the energy barrier of the reverse dissociation of triuret to CA $+\mathrm{CI}$. As the number of decomposition pathways of triuret is very large, we only explored the ones similar to those of biuret that could produce iso-cyanuric acid. The most favoured unimolecular decomposition 
route of triuret involves its isomerization into its tautomer (TT) with a critical energy of 36 $\mathrm{kcal} / \mathrm{mol}$, which, in turn, decomposes into allophanamic isocyanate (AI) and $\mathrm{NH}_{3}$ with a critical energy of $50.4 \mathrm{kcal} / \mathrm{mol}$. The potential energy surface of triuret decomposition is given in Figure S4.

Figure 5 presents the potential energy surface leading to the formation of isocyanuric acid (ICA) from AI.

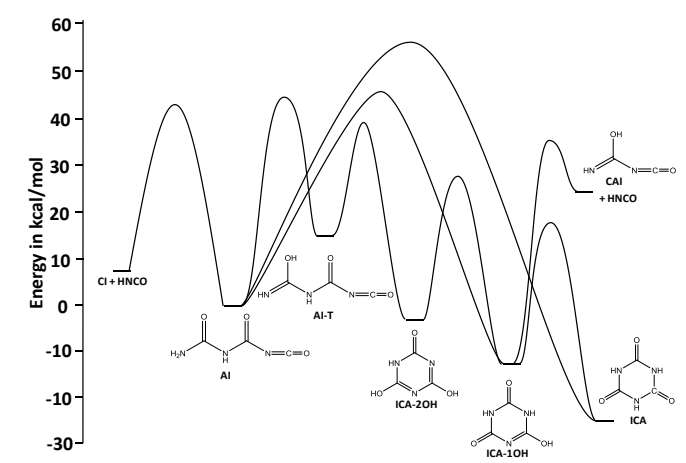

Figure 5: Potential energy surface of the unimolecular decomposition of allophanamic isocyanate (AI). Energy at $0 \mathrm{~K}$ computed at the CBS-QB3 level of theory.

The direct cyclization of AI into ICA is possible but involves an energy barrier that lies 13 $\mathrm{kcal} / \mathrm{mol}$ above the dissociation pathway to $\mathrm{CI}$ and $\mathrm{HNCO}$. The other possible direct cyclization yields a tautomer of ICA (ICA-1OH) with a critical energy of $46.7 \mathrm{kcal} / \mathrm{mol}$. Indirect cyclization of AI involves an intermediate isomerization step to AI-T $(44.9 \mathrm{kcal} / \mathrm{mol}$ energy barrier), followed by a favourable cyclization to ICA-2OH tautomer. ICA formation from its tautomer appears to be an easy process from a potential energy point of view. Figure 5 also shows that the reaction of CAI with $\mathrm{HNCO}$ can yield ICA-1OH which, in turn, can produce ICA.

Two unimolecular decomposition reactions were considered for carbamic acid:

$$
\begin{aligned}
& \mathrm{NH}_{2} \mathrm{COOH} \leftrightarrows \mathrm{NH}_{3}+\mathrm{CO}_{2} \\
& \mathrm{NH}_{2} \mathrm{COOH} \leftrightarrows \mathrm{HNCO}+\mathrm{H}_{2} \mathrm{O}
\end{aligned}
$$

The decarboxylation (R14) is the most favoured pathway with an energy barrier of 42.8 $\mathrm{kcal} / \mathrm{mol}$, which is $13 \mathrm{kcal} / \mathrm{mol}$ below the water elimination barrier (R15) at the CBS-QB3 level of calculation. 
Since the formation of carbamic acid is accompanied by the production of $\mathrm{HNCNH}$, we also considered the possible molecular growth pathways of carbodiimide by its reactions with itself and HNCO. These reactions are presented in Table S3. It can be noted that the final products of the successive possible additions are melamine, amelide and ameline, which are the minor products of urea pyrolysis.

\section{Simulations of urea pyrolysis}

The final urea pyrolysis mechanism includes urea unimolecular reactions (Figure 1) and the subsequent reactions leading to triuret. Figure S5 summarizes the triuret formation route on a potential energy surface. Triuret decomposition to AI (Figure S4) and its cyclizations to ICA (Figure 5) as well as the formation and decomposition of CC (summarized in Figure S6) are also included in the final kinetic model. Thermochemical and kinetic data used in the model are calculated at the CCSD(T)/CBS//B2PLYP-D3/cc-pVTZ level of theory with internal rotor treatments and Eckart tunnelling. Transition state theory was applied for all reaction pathways, except for the one involving pre-reactive complexes. For these cases, the twotransition-states model implemented in the MESS code was used [18]. The potential describing the barrierless formation of the VdW complexes was fitted on B2PLYP-D3/ccpVTZ relaxed scans between the centers of mass of the fragments.

Figure 6 presents the pyrolysis of pure urea at $1 \mathrm{~atm}$, in a constant pressure and temperature homogeneous batch reactor.

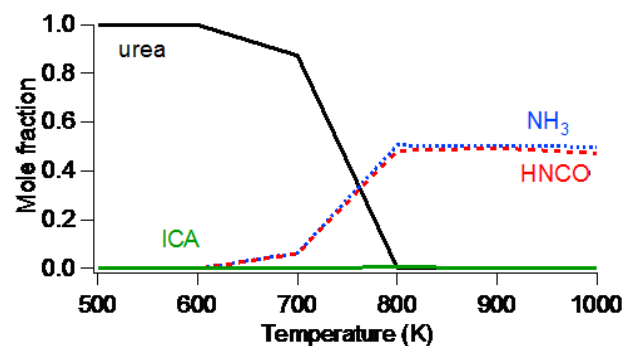

Figure 6: Simulation of the pyrolysis of pure urea at $1 \mathrm{~atm}$, for a residence time of 10s. Homogeneous batch reactor at constant pressure and temperature. 
The simulations are performed for a residence time of $10 \mathrm{~s}$, which corresponds to the same order of magnitude that the induction time for gas phase chemistry (between $773 \mathrm{~K}$ and 873 K) reported by Stein et al. [3] in their decomposition model of urea-water solution droplets. Under the conditions of Figure 6, urea decomposition starts at $600 \mathrm{~K}$ and its total consumption is achieved at $800 \mathrm{~K}$. The pyrolysis products are $\mathrm{NH} 3$ and $\mathrm{HNCO}$. The model does not predict any other by-product. A small fraction of isocyanuric acid (ICA) is found in the simulations, with a maximum mole fraction of $0.7 \%$ at $800 \mathrm{~K}$. An increase of pressure favours the formation of ICA. At 10 atm and $800 \mathrm{~K}$, its mole fraction is $6.1 \%$ and $16.6 \%$ at $50 \mathrm{~atm}$. Formation of by-products of urea the pyrolysis experiments is favoured for high-pressure conditions. We made exploratory simulations to model a condensed phase system, by increasing the pressure. In this approximate approach, a pressure of 1000 atm was chosen. These simulations were qualitatively compared to experimental data measured in an open reaction vessel by Schaber et al.[4] who reported the mass of urea, biuret and isocyanuric acid at different temperatures. In this experiment, a known mass of solid urea was heated to the desired temperature, and the solid/liquid residue was analysed with HPLC. For comparisons, the experimental masses of urea, biuret and ICA were converted to their mole equivalent and mole fractions were calculated, at each temperature, for this 3-compounds system. The same approach was used with the simulations performed at 1000 atm: the total number of moles in the reactor was calculated from the ideal gas law and the mole fraction of the same 3compounds were determined as a function of temperature (500 to $1200 \mathrm{~K}$ ). Residence time was set to $10 \mathrm{~min}$ as the experimental heating time is unknown.

Figure 7 presents the comparison between the experimental and simulated 3-compounds mole fraction. Since the experimental system is heterogeneous and our simulations are for an ideal gas the temperatures of simulations are shifted by $180 \mathrm{~K}$ to match the conversion of urea. Note that increasing the residence time would decrease the temperature shift. This analysis is therefore purely qualitative. 


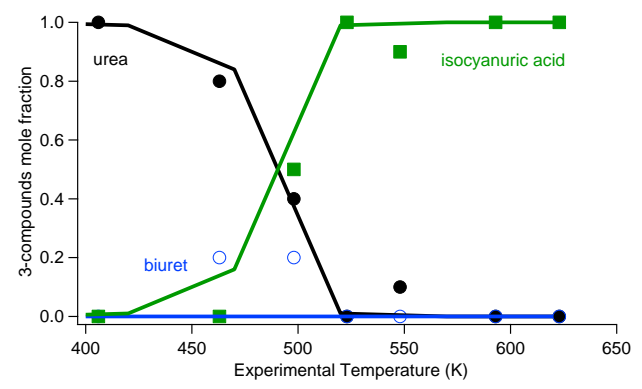

Figure 7: Pyrolysis of urea in an open reaction vessel. Symbols: experimental data from Schaber et al.[4] converted to mole fractions (see text). Lines: simulated product yields at 1000 atm and 10 min residence time. Simulated temperatures are shifted by $180 \mathrm{~K}$.

The simulations are able to capture the trends in selectivity despite the different state of matters. A small peak of biurea is observed at early urea conversion in both experiments and simulations. Analyses of the simulation results show that isocyanuric acid formation route follows the lowest energy pathway proposed in this work: formation of biuret from CA + $\mathrm{HNCO}$, biuret decomposition into $\mathrm{CI}+\mathrm{NH} 3$, formation of triuret from $\mathrm{CI}+\mathrm{CA}$, and triuret decomposition and cyclization into ICA. Carbamimidic acid (CA) is shown to play a central role in the growth of urea pyrolysis by-products. The hypotheses of the literature on the byproducts formation in urea pyrolysis pathways are shown to be unlikely. Only molecular reactions were investigated here. If no radical mechanism is possible at such temperature because of high bond dissociation energies of urea, $\mathrm{CA}, \mathrm{NH} 3$ and $\mathrm{HNCO}$, the role of ionic processes need to be explore in future works.

\section{Conclusions}

In this work, a systematic theoretical study of the pyrolysis of urea was performed, with a focus on the growth of known by-products of the literature. Electronic structure calculations demonstrated that the initial unimolecular decomposition of urea can lead to the formation of $\mathrm{NH}_{3}$ and $\mathrm{HNCO}$ through a direct pathway or via the formation of carbamimidic acid. This intermediate is found to promote the formation of heavier products (biuret and triuret), through the formation of stable pre-reactive complexes with HNCO and carbamic isocyanate. The latter compound and allophanamic isocyanate are produced from biuret and triuret. The 
pathway proposed here to explain the formation of isocyanuric acid, has never been envisaged in the literature. Simulations performed with a detailed kinetic model showed that the formation of isocyanuric acid is small in the gas phase and that its branching ratio increases with pressure. Qualitative simulations of a condensed phase were able to reproduce the branching ratio of experiments on urea, but with a shift in temperature was applied to fill the gap between gas phase and the heterogeneous phase experiments and possible ionic or catalytic processes. Future works on these alternative elementary mechanisms are necessary.

\section{Supplemental material}

The final and trial detailed chemical kinetic models are available as supplemental material. Tables describing all the possible reactions as well as supplementary potential energy surfaces and results of the trial simulations are also given.

\section{Acknowledgements}

High performance computing resources were partially provided by the EXPLOR center, hosted by the University of Lorraine. This work was also granted access to the HPC resources of IDRIS under the allocation 2018-A0010807249 made by GENCI. Dr S. J. Klippenstein is greatfully acknowledged for his help with the MESS code. 


\section{References}

[1] K. Skalska, J.S. Miller, S. Ledakowicz, Trends in NOx abatement: A review, Science of the total environment 408 (2010) 3976-3989.

[2] S. Tischer, M. Börnhorst, J. Amsler, G. Schoch, O. Deutschmann, Thermodynamics and reaction mechanism of urea decomposition, Physical Chemistry Chemical Physics 21 (2019) 16785-16797.

[3] M. Stein, V. Bykov, A.B. Abai, C. Janzer, U. Maas, O. Deutschmann, M. Olzmann, A reduced model for the evaporation and decomposition of urea-water solution droplets, International Journal of Heat and Fluid Flow 70 (2018) 216-225.

[4] P.M. Schaber, J. Colson, S. Higgins, D. Thielen, B. Anspach, J. Brauer, Thermal decomposition (pyrolysis) of urea in an open reaction vessel, Thermochimica acta 424 (2004) 131-142.

[5] M. Eichelbaum, R.J. Farrauto, M.J. Castaldi, The impact of urea on the performance of metal exchanged zeolites for the selective catalytic reduction of NOx: Part I. Pyrolysis and hydrolysis of urea over zeolite catalysts, Applied Catalysis B: Environmental 97 (2010) 9097.

[6] A.M. Bernhard, D. Peitz, M. Elsener, A. Wokaun, O. Kröcher, Hydrolysis and thermolysis of urea and its decomposition byproducts biuret, cyanuric acid and melamine over anatase TiO2, Applied Catalysis B: Environmental 115 (2012) 129-137.

[7] W. Brack, B. Heine, F. Birkhold, M. Kruse, G. Schoch, S. Tischer, O. Deutschmann, Kinetic modeling of urea decomposition based on systematic thermogravimetric analyses of urea and its most important by-products, Chemical Engineering Science 106 (2014) 1-8.

[8] D. Wang, N. Dong, S. Hui, Y. Niu, Analysis of urea pyrolysis in 132.5-190 C, Fuel 242 (2019) 62-67.

[9] K. Huthmacher, D. Most, Cyanuric acid and cyanuric chloride, Ullmann's Encyclopedia of Industrial Chemistry (2000).

[10] A. Nicolle, S. Cagnina, T. De Bruin, First-principle based modeling of urea decomposition kinetics in aqueous solutions, Chemical Physics Letters 664 (2016) 149-153.

[11] D. Gratzfeld, M. Olzmann, Gas-phase standard enthalpies of formation of urea-derived compounds: A quantum-chemical study, Chemical Physics Letters 679 (2017) 219-224.

[12] A. Jalan, R.H. West, W.H. Green, An extensible framework for capturing solvent effects in computer generated kinetic models, The Journal of Physical Chemistry B 117 (2013) 29552970.

[13] E. Moine, R. Privat, J.-N. Jaubert, B. Sirjean, N. Novak, E. Voutsas, C. Boukouvalas, Can we safely predict solvation Gibbs energies of pure and mixed solutes with a cubic equation of state?, Pure and Applied Chemistry (2019).

[14] J. Lizardo-Huerta, B. Sirjean, R. Bounaceur, R. Fournet, Intramolecular effects on the kinetics of unimolecular reactions of $\beta$-HOROO and $\mathrm{HOQ}^{\circ} \mathrm{OOH}$ radicals, Physical Chemistry Chemical Physics 18 (2016) 12231-12251.

[15] R. CHEMKIN-PRO, 15112, Reaction Design, Inc., San Diego, CA (2011).

[16] J.A. Miller, S.J. Klippenstein, The recombination of propargyl radicals and other reactions on a C6H6 potential, The Journal of Physical Chemistry A 107 (2003) 7783-7799.

[17] P. Glarborg, J.A. Miller, B. Ruscic, S.J. Klippenstein, Modeling nitrogen chemistry in combustion, Progress in Energy and Combustion Science 67 (2018) 31-68.

[18] Y. Georgievskii, J.A. Miller, M.P. Burke, S.J. Klippenstein, Reformulation and solution of the master equation for multiple-well chemical reactions, The Journal of Physical Chemistry A 117 (2013) 12146-12154. 